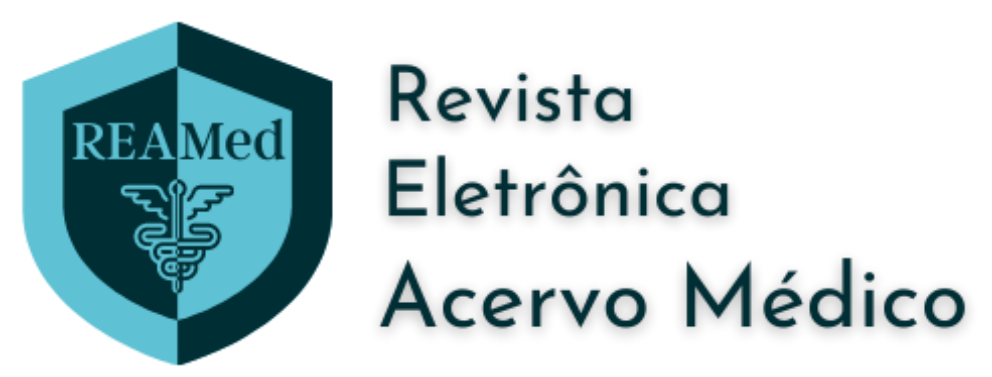

\title{
O uso de antiagregantes plaquetários na pré-eclâmpsia: uma revisão narrativa
}

The use of antiplatelet agents in preeclampsia: a narrative review

El uso de agentes antiplaquetarios en la preeclampsia: una revisión narrativa

Mariana Cardoso Bertoco ${ }^{1 *}$, Kamylla Santos Azambuja1.

\section{RESUMO}

Objetivo: Discorrer sobre a pré-eclâmpsia em gestantes e o uso de antiagregantes plaquetários como prevenção desta doença de forma atualizada. Revisão bibliográfica: A pré-eclâmpsia é uma condição relacionada à gravidez que pode ser fatal para mãe e feto. É uma das principais causas de morbimortalidade materna no Brasil. Caracteriza-se por pressão arterial alta, edema significativo e proteinúria. O uso de AAS, um antiagregante plaquetário, em pacientes com alto risco ou que já tiveram pré-eclâmpsia anteriormente se mostrou benéfico e seguro em prevenir a doença nos estudos avaliados. Considerações finais: $O$ uso de AAS em baixas doses em pacientes com risco de desenvolver pré-eclâmpsia apresenta boas evidências, entretanto, ainda não há consenso mundial sobre doses, quando iniciar o tratamento ou como fazer o rastreamento adequado nas gestantes. Há ainda a necessidade de encontrar novas terapias para a doença e melhorar o desfecho materno e fetal em pacientes que desenvolveram a PE mesmo após o uso de AAS.

Palavras-chave: Gestação, Antiagregantes plaquetários, Aspirina, Pré-eclâmpsia.

\section{ABSTRACT}

Objective: To discuss preeclampsia in pregnant women and the use of antiplatelet agents to prevent this disease in an updated way. Bibliographic review: Preeclampsia is a pregnancy-related condition that can be fatal for both mother and fetus. It is one of the main causes of maternal morbidity and mortality in Brazil. It is characterized by high blood pressure, significant edema, and proteinuria. The use of ASA, an antiplatelet agent, in patients at high risk or who had previously had preeclampsia was shown to be beneficial and safe in preventing the disease in the studies evaluated. Final considerations: The use of ASA at low doses in patients at risk of developing preeclampsia shows good evidence, however, there is still no global consensus on doses, when to start treatment or how to properly screen pregnant women. There is still a need to find new therapies for the disease and improve maternal and fetal outcomes in patients who developed PE even after using ASA.

Key words: Pregnancy, Antiplatelet agents, Aspirin, Preeclampsia.

\footnotetext{
1 Hospital Geral de Cuiabá, Cuiabá - MT.

*E-mail: maribertoco@gmail.com
} 


\section{RESUMEN}

Objetivo: Discutir la preeclampsia en gestantes y el uso de antiagregantes plaquetarios para prevenir esta enfermedad de forma actualizada. Revisión bibliográfica: La preeclampsia es una condición relacionada con el embarazo que puede ser fatal tanto para la madre como para el feto. Es una de las principales causas de morbilidad y mortalidad materna en Brasil. Se caracteriza por hipertensión arterial, edema importante y proteinuria. El uso de AAS, un agente antiplaquetario, en pacientes de alto riesgo o que previamente habían tenido preeclampsia se mostró beneficioso y seguro en la prevención de la enfermedad en los estudios evaluados. Consideraciones finales: El uso de AAS en dosis bajas en pacientes con riesgo de desarrollar preeclampsia muestra buena evidencia, sin embargo, aún no existe un consenso mundial sobre las dosis, cuándo iniciar el tratamiento o cómo realizar un tamizaje adecuado a las embarazadas. Todavía existe la necesidad de encontrar nuevas terapias para la enfermedad y mejorar los resultados maternos y fetales en pacientes que desarrollaron EP incluso después de usar AAS.

Palabras clave: Embarazo, Agentes antiplaquetarios, Aspirina, Preeclampsia.

\section{INTRODUÇÃO}

A gestação é um momento representativo para a família num contexto social e de realização pessoal. № entanto, algumas alterações na saúde materna podem aumentar o risco de um desfecho inesperado tanto para a mãe, quanto para o feto. A pré-eclâmpsia representa uma das principais causas de morte materna no mundo (MACHADO N, 2020).

Entre os anos de 2010 a 2017 ocorreram 8992 mortes por causas obstétricas diretas no Brasil. Destas, a pré-eclâmpsia ocupa o primeiro lugar, com cerca de 1264 óbitos, o que representa 14,06\%. No estudo considerado, foi evidenciado ainda que estas mortes se concentram principalmente nas regiões Sudeste e Nordeste do País. A faixa etária mais acometida foi a de mulheres entre 20 e 29 anos $(41,30 \%)$ e 30 a 39 anos (40,13\%) (GOMES TB, et al., 2020).

A pré-eclâmpsia é uma condição relacionada à gravidez que pode ser fatal para a mãe e o feto. Caracteriza-se por pressão arterial alta, edema significativo e perda de proteína na urina. A pré-eclâmpsia ocorre durante a segunda metade da gravidez, a partir de 20 semanas de amenorreia. A pré-eclâmpsia recebe esse nome pelo fato de poder levar a uma crise eclâmptica, uma complicação séria que resulta em convulsões, muito parecidas com uma crise epilética (KAHHALE S, et al., 2018).

A pré-eclâmpsia pode ter outras complicações graves que às vezes justificam a internação da paciente até o nascimento. Ela termina com o nascimento da criança e a expulsão da placenta. Todos os tratamentos médicos implementados durante a pré-eclâmpsia servem para manter a gravidez até o termo, ou, até uma idade gestacional compatível com a sobrevivência do feto, geralmente após 24 semanas de gestação. Após o parto, os sintomas desaparecem em poucos dias na maioria dos casos (BRAGA JC, et al., 2021).

A pré-eclâmpsia, por se tratar de uma doença hipertensiva, nem sempre causa sintomas. A hipertensão arterial associada pode causar inchaço das mãos, pés e face em particular, embora sua presença por si só não comprove a existência de pré-eclâmpsia. Quando a pré-eclâmpsia é grave, a paciente também pode ter dores de cabeça persistentes, zumbidos nos ouvidos, manchas escuras ou brilhantes que se movem no campo de visão ou dor intensa logo abaixo das costelas, especialmente do lado direito (KAHHALE S, et al., 2018).

O papel da aspirina na Pré-eclâmpsia é estudado desde 1978, com a descrição de um estudo onde uma paciente, que apresentava a doença de forma recorrente, parece ter se beneficiado com o uso da aspirina. Ainda assim, até hoje, após inúmeros estudos e publicações, não há um protocolo padrão bem definido sobre a dosagem, quando iniciar e como rastrear a população de alto risco de forma abrangente. Existem estudos que sugerem ainda que os benefícios podem não se aplicar a gestantes previamente hipertensas (HUAl J, et al., 2021). 
A aspirina é um dos fármacos mais utilizados no mundo todo. Possui um papel analgésico, antipirético e atua na profilaxia cardiovascular. Estudos demonstraram que a aspirina pode prevenir algumas desordens relacionadas à gestação, como a pré-eclâmpsia, prematuridade e crescimento fetal restrito. Este fato se dá devido suas características anti-inflamatórias e antitrombóticas (LIN L, et al., 2018).

As indicações para o uso de aspirina durante a gestação ainda são um tema de muito debate entre os especialistas e diferem entre os países. Os estudos demonstram ef etividade na prevenção secundária em pacientes que já tiveram pré-eclâmpsia em gestações anteriores. Por outro lado, em pacientes que nunca tiveram o diagnóstico a eficácia é bastante controversa. A grande maioria dos estudos referem-se a pessoas brancas e negras, enquanto faltam dados suficientes em outras populações, como as asiáticas (ATALLAH A, et al., 2017).

Assim, o objetivo geral desta pesquisa foi apresentar, por meio de uma revisão narrativa atualizada, a importância do uso de antiagregantes plaquetários na pré-eclâmpsia, uma doença grave e prevalente entre gestantes. Os objetivos específicos buscam apresentar e conceituar o que é a pré-eclâmpsia, bem como destacar e conceituar o que são os antiagregantes e os objetivos com seu uso durante a gestação.

\section{REVISÃO BIBLIOGRÁFICA}

\section{A pré-eclâmpsia}

A pré-eclâmpsia refere-se a uma doença hipertensiva que pode complicar a gravidez (hipertensão da gravidez). Em casos raros, pode ocorre até 14 dias após o parto. No Brasil, ela é considerada a maior causa de morbimortalidade entre mulheres em período fértil. A pré-eclâmpsia é tradicionalmente caracterizada pelos principais sintomas de hipertensão e proteinúria (proteína na urina). O edema (retenção hídrica) por si só não af eta o prognóstico da mãe e da criança, sendo considerado normal na gestação mesmo sem a presença da pré-eclâmpsia (MACHADO NC, 2020).

Além dos principais sintomas citados (pressão alta, proteinúria e edema), os acometidos relatam tonturas e dores de cabeça, sonolência, distúrbios visuais como olhos piscando, além de náuseas e vômitos. O mé dico pode notar hiperreflexia (reflexos aumentados). Além disso, em $20 \%$ dos casos há envolvimento hepático e, portanto, aumento dos valores hepáticos, o que pode ser demonstrado em exames laboratoriais (BRAGA JC, et al., 2021).

As causas da pré-eclâmpsia não são claramente compreendidas. A implantação perturbada do trofoblasto é discutida, o que significa que os vasos sanguíneos na decídua não são remodelados e expandidos como é realmente necessário durante a gravidez. Apoiar esta hipótese é que a diamina oxidase secretada por trofoblastos extravilosos na corrente sanguínea materna é significativamente diminuída no início da gravidez naquelas mulheres que foram diagnosticadas posteriormente com pré-eclâmpsia (MACHADO NC, 2020).

Também distúrbios no metabolismo das prostaglandinas parecem desempenhar um papel. No entanto, uma origem bacteriana ou viral é improvável. Se houver suspeita de pré-eclâmpsia a gestante relata tonturas e dores de cabeça, sonolência, visão turva, náuseas e vômitos. Para o diagnóstico, várias medidas de pressão arterial devem ser feitas primeiro (MAI CM, et al., 2021).

A excreção de proteínas na urina (proteinúria) deve ser medida, não com tiras reagentes de urina para o diagnóstico, pois as gestantes também excretam proteínas aumentadas fisiologicamente, mas com coleta de urina de 24 horas. As tiras de teste de urina podem ser usadas para monitorar o progresso. Embora o edema tenha perdido importância diagnóstica, ele pode ser usado para um acompanhamento aproximado pela medição do peso (MACHADO NC, 2020).

A única terapia causal é a interrupção prematura da gravidez. Após o nascimento, a condição da mãe geralmente melhora rapidamente. No entanto, como uma mulher mais velha, ela tem um risco muito alto de desenvolver pressão alta novamente (BRAGA JC, et al., 2021).

Como a causa da doença ainda não está clara, deve-se ter cuidado ao tratar os sintomas. Em particular, a tentativa de combater o edema com uma dieta pobre em sal ou mesmo curas de desidratação geralmente 
leva a uma deterioração do estado de saúde da gestante e a uma condição ameaçadora do feto, que muitas vezes só pode ser salva por um cesariana de emergência imediata (MACHADO NC, 2020).

Crianças nascidas de semanas a meses prematuras muitas vezes lutam com hemorragia cerebral, síndrome do desconforto respiratório, insuficiência renal ou danos oculares e podem permanecer com problemas de desenvolvimento ao longo da vida (KAHHALE S, et al., 2018).

Uma diminuição descontrolada da pressão arterial com medicação pode levar a uma subalimentação do feto e, portanto, só deve ser realizada para proteger a gestante quando os valores estiverem constantemente acima de 170/110 mmHg. No entanto, a pressão arterial não deve ser reduzida abaixo de 140/90 mmHg para não reduzir demais a "pressão alta necessária " da criança e, assim, não colocar a criança em risco (KAHHALE S, et al., 2018).

No entanto, preparações especiais podem estar dilatando os vasos uterinos e conseguir uma redução ef etiva da pressão arterial, que é necessária para a mãe e, ao mesmo tempo, continuar a garantir os cuidados da criança. Devido à perda de proteína devido à proteinúria, proteína suficiente deve ser fornecida através da dieta (BRAGA JC, et al., 2021).

A monitorização regular dos batimentos cardíacos da criança com o cardiotocograma, bem como o crescimento regular e, se necessário, os exames Doppler da criança são obrigatórios para diagnosticar atempadamente a insuficiência placentária crónica. Em casos extremos, a interrupção prematura da gravidez é inevitável para evitar a eclampsia, que pode ser fatal tanto para a criança quanto para a mãe (MACHADO NC, 2020).

A prevenção primária da pré-eclâmpsia é feita com o uso de baixas doses de aspirina (50-150mg diárias), com estudos corroborando seu uso em gestantes em alto risco para o desenvolvimento da doença ou que já tiveram a pré-eclâmpsia em gestações anteriores (ATALLAH A, et al., 2017). Um estudo considerando o uso de quercetina, um bioflavonóide com propriedades antioxidantes e renoprotetoras, em suplementação à aspirina na prevenção da pré-eclâmpsia se mostrou promissor em ratos, aumentando os ef eitos terapêuticos da aspirina. De fato, ainda há poucas estratégias terapêuticas para a pré-eclâmpsia, acarretando um grande problema de saúde (YANG S, et al., 2019).

Estudos considerando novas alternativas terapêuticas, como o uso de anestésico epidural na tentativa de obter controle pressórico e dos demais sintomas da pré-eclâmpsia foram conduzidos, porém ainda carecem de evidências robustas para analisar a ef etividade e segurança de tais medidas (RAY A e RAY S, 2017).

\section{Antiagretante plaquetário}

Um agente antiagregante ou antiplaquetário, é uma droga que diminui a agregação plaquetária e inibe a formação de trombos. Os antiagregantes são eficazes na circulação arterial, onde os anticoagulantes têm pouco ef eito. Eles são frequentemente usados na prevenção primária e secundária de doenças trombóticas cerebrovasculares ou cardiovasculares, como a síndrome coronariana aguda, uma complicação trombótica do ateroma da artéria coronária (REIS-DE-CARVALHO C, 2021).

Eles podem ser administrados isoladamente, mas os diferentes mecanismos de ação significam que muitas vezes são sinérgicos e às vezes prescritos em combinação. Eles são administrados principalmente para prevenção secundária (ou seja, após um primeiro acidente) de uma complicação do ateroma: doença coronariana (angina pectoris, infarto do miocárdio), acidente vascular cerebral, arteriopatia obliterante dos membros inferiores, pré-eclâmpsia entre outras. Eles também podem ser administrados na prevenção da formação de trombose intracardíaca (coágulo) durante a fibrilação atrial, embora sejam menos eficazes do que um anticoagulante oral (DULEY L, 2021).

Todos os antiagregantes não apresentam resultados equivalentes e a escolha de um deles é baseada na comprovação de sua eficácia na doença alvo, na via desejada (oral ou intravenosa), na tolerância, na homogeneidade dos resultados, avaliação do risco hemorrágico e custo. Este último fator não é desprezível, pois a prescrição na maioria das vezes é feita "para toda a vida". No Brasil, em termos de disponibilidade, o agente mais usado é o Ácido Acetilsalicílico (AAS) (SERRANO CV, 2019). 
Além dos riscos específicos de cada molécula, eles aumentam o risco de sangramento, seja espontâneo ou durante uma operação. A combinação com anticoagulantes deve ser particularmente cautelosa. A terapia dupla também aumenta o risco (DULEY L, 2021).

Em caso de cirurgia, a interrupção dos antiagregantes uma semana antes minimiza o risco de sangramento, mas aumenta muito significativamente o risco de ocorrência de eventos trombóticos que podem ser muito graves. De fato, a maioria das operações pode ser feita com pequenas doses de aspirina sem grande risco de sangramento e não é recomendado interromper este último (REIS-DE-CARVALHO C, 2021). No caso de terapia dupla, deve-se decidir entre adiar a intervenção até que ela possa ser alterada para monoterapia ou interromper um dos dois antiagregantes, o que não é recomendado no caso de procedimento de angioplastia com colocação de stent muito recente (SERRANO CV, 2019).

Os efeitos dos agentes antiplaquetários foram avaliados pela primeira vez em pequenos ensaios randomizados, que relataram reduções marcantes no risco de hipertensão e proteinúria. Esses estudos eram muito pequenos para fornecer informações confiáveis sobre outros resultados mais substantivos, como mortalidade perinatal e parto prematuro. Além disso, não havia informações sobre os riscos potenciais da terapia antiplaquetária, como um possível aumento do risco de sangramento tanto para a mulher quanto para o bebê, ou possíveis ef eitos no desenvolvimento do bebê e da criança (MIRABITO COLAFELLA KM, et al., 2020).

Os resultados promissores desses primeiros ensaios levaram a vários grandes estudos em todo o mundo. Antes que isso pudesse ser concluído, no entanto, o uso de aspirina em baixas doses já havia se tornado relativamente difundido para mulheres consideradas com risco aumentado de pré-eclâmpsia. Os resultados dos estudos maiores foram decepcionantes, pois não conseguiram confirmar nenhuma redução estatisticamente significativa nos resultados substantivos (DULEY L, et al., 2019).

A prescrição da terapia antiplaquetária deve ser individualizada com base nos fatores de risco (na melhor das hipóteses avaliados por uma escala de risco) e nas características clínicas (risco de sangramento, etc.) dos pacientes, bem como nas propriedades farmacológicas, respectivas indicações, tolerância e custo da agentes antiplaquetários (DULEY L, et al., 2019).

Como visto, a pré-eclâmpsia é uma das principais causas de mortalidade e morbidade durante a gravidez e o parto. Agentes antiplaquetários, especialmente aspirina (AAS) em baixas doses, podem prevenir ou retardar a pré-eclâmpsia e, assim, melhorar o resultado. Corroborando com esta ideia, a autora Atallah $A$, et al., (2017) salienta que a aspirina também é amplamente utilizada para prevenir distúrbios cardiovasculares relacionados à gravidez, como pré-eclâmpsia e restrição de crescimento intrauterino, e distúrbios maternos como síndrome antifosfolípide.

A aspirina, ou ácido acetilsalicílico, é um agente antiagregante plaquetário inibidor não seletivo e irreversível da enzima ciclooxigenase (COX), com propriedades antiplaquetárias e anti-inflamatórias. Normalmente a COX produz prostaglandinas, a maioria das quais são pró-inflamatórias (causam inflamação), e tromboxanos, que promovem a coagulação (MA'AYEH M, et al., 2020). Recomenda-se o tratamento preventivo de gestantes com alto risco de pré-eclâmpsia (grau A) com aspirina em baixa dose $(75 \mathrm{mg} / \mathrm{d})$. Este tratamento deve ser iniciado antes da 16 ${ }^{\mathrm{a}}$ semana (grau A), mas parece desejável interrompê-lo antes da $32^{\mathrm{a}}$ semana (consenso profissional) (ROLNIK D, et al., 2017).

Pacientes com alto risco de retardo de crescimento intrauterino se beneficiam do mesmo tratamento. Não é recomendado dar aspirina para evitar abortos repetidos inexplicáveis (grau A). Em caso de abortos espontâneos precoces repetidos $(n \geq 3)$ ou aborto tardio associado à síndrome antifosfol ípede (e na ausência de trombose venosa ou arterial), recomenda-se tratar o pré-parto ou antes da $6^{a}$ semana com dose aspirina (75 mg/d) em combinação com heparina (grau A). No caso de fertilização in vitro, a aspirina não deve ser usada na prática atual para aumentar a taxa de sucesso (grau B). Indicações não obstétricas para aspirina em mulheres grávidas devem ser avaliadas (ROBERGE S, et al., 2018).

A aspirina (75-100 $\mathrm{mg} / \mathrm{dia})$ é recomendada em combinação com heparina para gestantes com válvula mecânica com alto risco de tromboembolismo (história de tromboembolismo, fibrilação atrial) (grau B) (HOFFMAN MK, et al., 2020). 
Por fim, a aspirina em prevenção secundária por motivos cardiológicos, neurológicos, vasculares ou reumatológicos pode ser mantida durante a gravidez. Recomenda-se a interrupção do tratamento a partir da $32^{\text {a }}$ semana de gestação devido ao risco de sangramento excessivo no momento do parto na mãe e fechamento prematuro do ducto arterial no feto (ROBERGE S, et al., 2018).

\section{CONSIDERAÇÕES FINAIS}

Apesar das boas evidências de que os agentes antiplaquetários, principalmente a aspirina em doses de 50-100mg/dia, reduzem a incidência de pré-eclâmpsia e suas consequências, como parto prematuro e mortalidade perinatal, permanece a incerteza sobre se algumas mulheres, em termos de risco, se beneficiam mais do que outras, em qual período da gestação o tratamento idealmente deve começar e se a eficácia do tratamento depende da dose antiplaquetária. O consenso parece ser que seu uso em gestantes que já tiveram Pré-eclâmpsia em gestações anteriores, ou que apresentam alto risco de desenvolver a doença, é altamente recomendado e seguro. Embora o estudo em gestantes seja dificultado por razões éticas e legais, ainda há muito espaço de pesquisa para elucidar melhor a forma ideal de conduzir o uso de antiagregantes na prevenção da pré-eclâmpsia, além da necessidade em encontrar novas terapias eficazes para seu tratamento, evitando desfechos ruins tanto para mãe quanto para o feto.

\section{REFERÊNCIAS}

1. ATALLAH A, et al. Aspirin for Prevention of Preeclampsia. Drugs, 2017;77(17):1819-1831.

2. BASCHAT AA, et al. Maternal blood-pressure trends throughout pregnancy and development of pre-eclampsia in women receiving first-trim ester aspirin prophylaxis. Ultrasound Obstet Gynecol, 2018;52(6): 728-733.

3. BRAGA JC, et al. Gravidez na adolescência com o fator de risco para pré-eclâmpsia. Revisão sistemática da literatura. Revista Multidisciplinar da Saúde, 2021;3(2):37-49.

4. DULEY L, et al. Antiplatelet agents for preventing pre-eclampsia and its complications. Cochrane Database Syst Ver, 2019; (10): CD004659.

5. DULEY L. Antiagregantes plaquetários para prevenção da pré-eclâmpsia e suas complicações: uma Revisão Cochrane. JBMEDE-Jornal Brasileiro de Medicina de Ëmergência, 2021;1 (2): e21016-e21016.

6. EVERETT TR, et al. Pre-eclampsia: the Potential of GSNO Reductase Inhibitors. Curr Hypertens Rep, 2017;19(3): 20.

7. GOMES TB, et al. Pré-eclâmpsia: importante causa de óbitos maternos no Brasil entre os anos de 2010-2017. Braz. J. of Develop, 2020;6(10): 75496-75510

8. HAMULYÁK EN, et al. Aspirin or heparin or both for improving pregnancy outcomes in women with persistent antiphospholipid antibodies and recurrent pregnancy loss. Cochrane Database Syst Ver, 2020;5(5): CD012852.

9. HOFFMAN MK, et al. Low-dose aspirin for the prevention of preterm delivery in nulliparous women with a singleton pregnancy (ASPIRIN): a randomised, double-blind, placebo-controlled trial. Lancet. 2020;395(10220):285-293.

10. HUAl J, et al. Preventive effect of aspirin on preeclampsia in high-risk pregnant women with stage 1 hypertension. Journal of clinical hypertension (Greenwich, Conn.), 2021;23(5):1060-1067.

11. KAHHALE S, et al. Pré-eclâmpsia. Revista de Medicina, 2018;97(2):226-234.

12. LIN L, et al. APPEC Study Group. Low-dose aspirin in the prevention of pre-eclampsia in China (APPEC study): protocol for a multicentre randomized controlled trial. Trials, 2018;19(1): 608.

13. MA'AYEH M, et al. Novel Interventions for the Prevention of Preeclampsia. Current hypertension reports, 2020; 22(2): 17.

14. MACHADO NC. Pré-eclâmpsia na gravidez sob a ótica das mulheres da região noroeste do Estado do Rio Grande do Sul, Brasil. O Mundo da Saúde, 2020; 1(44):498-505.

15. MAI CM, et al. Assistência de enfermagem em mulheres com pré-eclâmpsia e/ou eclâmpsia: uma revisão integrativa da literatura. Boletim de Conjuntura (BOCA), 2021;8(23): 28-39.

16. MIRABITO COLAFELLA KM, et al. Aspirin for the prevention and treatment of pre-eclampsia: $A$ matter of COX-1 and/or COX-2 inhibition? Basic Clin Pharmacol Toxicol, 2020;127(2):132-141.

17. RAY A, RAY S. Epidural therapy for the treatment of severe pre-eclampsia in non labouring women. Cochrane Database Syst Ver, 2017;11(11): CD009540.

18. REIS-DE-CARVALHO C. Análise da Revisão Cochrane: O Papel dos Antiagregantes Plaquetários para Prevenir a Pré-Eclâmpsia e as Suas Complicações. Acta Medica Portuguesa, 2021;34:12.

19. ROBERGE S, et al. Meta-analysis on the effect of aspirin use for prevention of preeclampsia on placental abruption and antepartum hemorrhage. Am J Obstet Gynecol, 2018;218(5): 483-489

20. ROLNIK D, et al. Aspirin versus Placebo in Pregnancies at High Risk for Preterm Preeclam psia. N Engl J Med, 2017 ; 377(7):613-622. 21. SERRANO CV. Posicionamento sobre antiagregantes plaquetários e anticoagulantes em cardiologia-2019. Arquivos
Brasileiros de Cardiologia, 2019;113:111-134.

22. TOLCHER MC, et al. Low-dose aspirin for preeclampsia prevention: efficacy by ethnicity and race. Am J Obstet Gynecol MFM, 2020;2(4): 100184.

23. YANG S, et al. Ameliorative effects of pre-eclampsia by quercetin supplement to aspirin in a rat model induced by $L$ NAME. Biomed Pharmacother, 2019;116:108969. 